\title{
Microwave oven safety: a food safety consumer survey in Malaysia
}

\begin{abstract}
Microwave oven is an indispensable tool in every household. However, the proper usage of the microwave ovens was neglected and thus, causing food safety concerns, especially microbiological hazard in microwaved heated food. Therefore, a survey was initiated to gauge the consumer's knowledge of microwave oven safety concerning the food safety of reheated food. A total of 29 survey questions was designed that covered 5 demographic questions and 24 others on the knowledge and practice of microwave oven safety and food safety attitude. The survey was blasted out to 329 respondents via email. Overall, 189 (57.4\%) returned respondents demonstrated low level of knowledge of the microwave oven safety, which indicated improper education of the microwave oven despite knowing the norms on the microwave oven usage. In addition, consumers demonstrated low levels of practicing microwave oven safety. The outcome of the survey also showed consumer's neutral attitude towards food safety. There was no statistically significant association between the variables and demographic. The minimum knowledge of microwave oven and food safety shown by the consumers requires the attention to improve the food safety educational programs by including the knowledge on microwave oven safety for the betterment of the public health.
\end{abstract}

Keyword: Microwave oven safety; Reheating; Food safety; Consumer survey 